- Case Report

\title{
Superior Vena Cava Syndrome Due to Mediastinal Tuberculous Lymphadenitis
}

\author{
Jin Ho Jang, Doosoo Jeon, Yun Seong Kim, Woo Hyun Cho, Hye Ju Yeo* \\ Department of Internal Medicine, Pusan National University Yangsan Hospital, Pusan National University School of Medicine, Yangsan, Korea
}

Superior vena cava (SVC) syndrome refers to a medical emergency resulting from compression of the SVC. It requires early diagnosis and treatment, and is usually caused by malignant tumors; rarely, mediastinal tuberculous lymphadenitis can cause SVC syndrome. Here, we present a case study of an immunocompetent 61-year-old woman who presented with acute onset SVC syndrome and was diagnosed with tuberculous lymphadenitis on thoracotomy; the symptoms resolved with anti-tuberculosis therapy. This unusual case highlights the importance of the differential diagnosis in patients presenting with acute onset SVC syndrome; a timely diagnosis and appropriate treatment lead to complete recovery.

Keywords: Superior vena Cava Syndrome; Lymphadenitis; Tuberculosis; Thoracotomy 


\section{INTRODUCTION}

Superior vena cava (SVC) syndrome refers to a medical emergency resulting from compression of the SVC; this syndrome presents with a group of signs and symptoms, such as facial edema, dyspnea, and cyanosis. It requires early diagnosis and treatment, and is usually caused by malignant tumors; rarely, mediastinal tuberculous lymphadenitis can cause SVC syndrome. ${ }^{1-5)}$ Here, we present the case of an immunocompetent 61-year-old woman with acute onset SVC syndrome, who was diagnosed with tuberculous lymphadenitis on thoracotomy; the symptoms resolved with anti-tuberculosis therapy.

\section{CASE REPORT}

A 61-year-old non-smoking woman was admitted to the hospital with a 2-week history of facial edema, dry cough, and dyspnea on exertion. She was experiencing tachypnea and moderate respiratory distress; her vital signs were as follows: blood pressure 100/60 mm Hg, heart rate 110 beats per minute, respiratory rate 28 breaths per minute, and oxygen saturation $94 \%$ on room air. Physical examination revealed facial edema and distended non-pulsatile superficial veins over the neck and chest. There was no lymphadenopathy and the systemic examination was unremarkable. Routine laboratory test results were within normal ranges. A chest radiograph revealed marked widening of the mediastinum without any lung parenchymal lesions (Figure 1A). Computed tomography (CT) of the chest revealed enlargement of the right upper paratracheal (45 $\mathrm{mm}$ in diameter) and both lower paratracheal lymph nodes (right lower paratracheal: $20 \mathrm{~mm}$ in diameter, left lower paratracheal: $23 \mathrm{~mm}$ in diameter), which were compressing the SVC. Each lymph node showed marginal contrast enhancement with low central attenuation (Figure 1B, C). Positron emission tomography showed abnormal fluorodeoxyglucose uptake in the upper paratracheal (standardized uptake value [SUVmax]=16.1), lower paratracheal (SUVmax=14.4), and hilar (SUVmax=11.0) lymph nodes. Owing to the short history and SVC obstruction with mediastinal lymph nodes, the differential diagnosis was considered in the following order: lymphoma, tuberculosis, and bacterial infection. Initial acid-fast bacillus staining of the sputum as well as the results of blood culture were all negative. The patient underwent a median thoracotomy to establish a diagnosis and relieve the symptoms of SVC syndrome. During the operation, the enlarged paratracheal lymph nodes were removed; histopathology of a biopsy specimen revealed chronic granulomatous inflammation and central caseous necrosis consistent with tuberculosis (Figure 2). An acid-fast stain of the biopsy specimen was negative, but a nested polymerase chain reaction for Mycobacterium tuberculosis was positive. After the surgical diagnosis, we began anti-tuberculosis treatment (i.e., isoniazid, rifampicin, ethambutol, and pyrazinamide). The symptoms of SVC syndrome all improved during hospitalization. The patient completed a 9-month course of anti-tuberculosis treat-

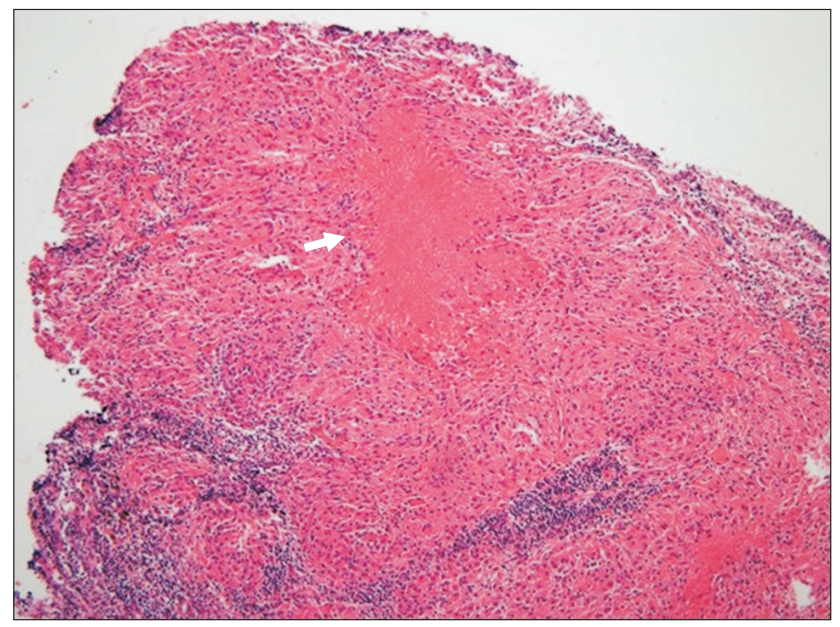

Figure 2. Histopathological findings of mediastinal lymph node: chronic granulomatous inflammation with central necrosis (indicated by white arrow) was seen.
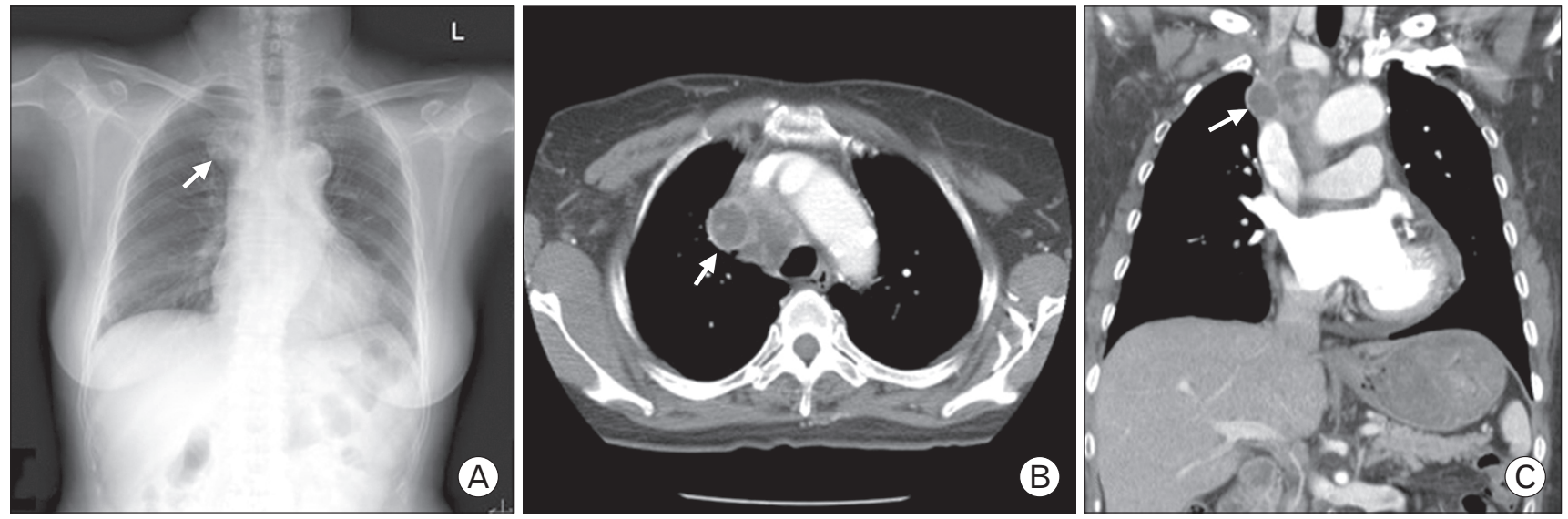

Figure 1. Radiologic findings of the chest. (A) Chest radiograph showed contour bulging (indicated by white arrow) at right mediastinum. (B) Computed tomography showed multiple mediastinal lymph nodes enlargement with central low density and peripheral rim enhancement (indicated by white arrow). (C) Coronal view of computed tomography revealed conglomerate mass encasing and compressing the superior vena cava (indicated by white arrow). 
ment and was in good condition at the 3-year follow-up.

\section{DISCUSSION}

Acute onset SVC syndrome is a critical disorder. In the pre-antibiotic era, complications of untreated infection such as syphilis and tuberculosis were frequent causes of SVC syndrome. ${ }^{6)}$ Subsequently, malignancy became the most common cause, accounting for $90 \%$ of cases by the $1980 \mathrm{~s}^{6,7)}$ More recently, the incidence of SVC syndrome due to thrombosis caused by intravascular devices has risen, and benign causes now account for $20 \%$ to $40 \%$ of cases of SVC syndrome. Tuberculosis is currently an uncommon cause of SVC syndrome. The rarity of SVC syndrome may cause delays in diagnosis and treatment, and inadequate diagnostic techniques can result in a substantial increase in morbidity and mortality.

In adults, tuberculous lymphadenitis is usually accompanied by lung parenchymal lesions; thus, it is difficult to discriminate between tuberculous lymphadenitis and other diseases, such as lung cancer, lymphoma, and sarcoidosis, in patients without parenchymal lesions. Flexible bronchoscopy has limited capability in the diagnosis of mediastinal tuberculous lymphadenitis, and usually invasive diagnostic techniques are needed. According to Khan et al., ${ }^{8}$ the rate of accurate diagnosis based on test type is: bronchoscopy $20 \%$, CT-guided fine needle aspiration $66 \%$, mediastinoscopy $75 \%$, and thoracotomy $100 \%$. Recently, Geake et al. ${ }^{9)}$ recommended endobronchial ultrasound transbronchial needle aspiration (EBUS-TBNA) as the first diagnostic procedure in patients with suspected mediastinal tuberculous lymphadenitis. Although EBUS-TBNA is a safe procedure and has a high yield, it is impractical under certain circumstances. The value of EBUSTBNA in the initial diagnosis of lymphoma remains controversial. Therefore, excisional biopsy is preferred in patients with suspected malignant SVC syndrome. In our case, the patient required prompt therapeutic intervention as well as tissue for diagnosis; thus, an urgent thoracotomy was performed to reduce extrinsic compression on the SVC, and resulted in immediate improvement. Previous case reports also utilized surgical diagnostic techniques, such as mediastinoscopy, ${ }^{3-5)}$ video-associated thoracoscopic surgery, ${ }^{1)}$ and thoracotomy; ${ }^{2)}$ these techniques were helpful in rapid differential diagnosis of malignancy and emergency management.

Mediastinal tuberculous lymphadenitis is treated with standard anti-tuberculosis medicine. All recently reported cases were cured using medical treatment alone without surgical intervention. ${ }^{1-5)}$ However, if SVC syndrome is present, surgery may be inevitable. Although medical treatment is needed as primary therapy, concomitant surgical biopsy and a debulking procedure may assist in the rapid diagnosis and prompt resolution of symptoms during an emergency. Owing to rarity of the case, there are no general guidelines regarding the role and indications for surgery in the treatment of mediastinal tuberculous lymphadenitis causing SVC syndrome. If the patient has severe, potentially life-threatening symptoms, such as cerebral edema, laryngeal edema, or lower blood pressure, surgical intervention may be required for immediate treatment of symptoms. Careful follow-up is required because symptoms with lymphadenopathy may be worsened due to a paradoxical reaction during anti-tuberculosis treatment. ${ }^{10)}$

In summary, this case highlights the importance of the differential diagnosis in patients presenting with acute onset SVC syndrome; a timely diagnosis and appropriate treatment lead to complete recovery.

\section{CONFLICT OF INTEREST}

No potential conflict of interest relevant to this article was reported.

\section{REFERENCES}

1. Kim SE, Kim CH, Park YB, Lee JY, Cho SJ, Shin HS, et al. Superior vena caval syndrome due to tuberculous lymphadenitis. Tuberc Respir Dis 2004;57:368-71.

2. Harbecke RG, Schlueter DP, Rosenzweig DY. Reversible superior vena caval syndrome due to tuberculosis. Thorax 1979;34:410-1.

3. Lee N, Lui G, Wong KT, Lam R, Cockram CS. Reversible superior vena cava obstruction caused by tuberculous lymphadenitis. QJM 2008;101:987.

4. Kumar A, Khilnani GC, Kumar S, Sharma SK. Acute superior vena cava obstruction due to tubercular mediastinal abscess. Indian J Chest Dis Allied Sci 2007;49:165-7.

5. Zisis C, Skevis K, Kefaloyannis EM, Avgoustou K, Bellenis I. Mediastinal tuberculous lymphadenitis presenting as superior vena cava syndrome. J Thorac Cardiovasc Surg 2006;131:e11-2.

6. Wilson LD, Detterbeck FC, Yahalom J. Clinical practice: superior vena cava syndrome with malignant causes. N Engl J Med 2007;356:1862-9.

7. Rice TW, Rodriguez RM, Light RW. The superior vena cava syndrome: clinical characteristics and evolving etiology. Medicine (Baltimore) 2006;85:37-42.

8. Khan J, Akhtar M, von Sinner WN, Bouchama A, Bazarbashi M. CTguided fine needle aspiration biopsy in the diagnosis of mediastinal tuberculosis. Chest 1994;106:1329-32.

9. Geake J, Hammerschlag G, Nguyen P, Wallbridge P, Jenkin GA, Korman TM, et al. Utility of EBUS-TBNA for diagnosis of mediastinal tuberculous lymphadenitis: a multicentre Australian experience. J Thorac Dis 2015;7:439-48.

10. Minguez C, Roca B, Gonzalez-Mino C, Simon E. Superior vena cava syndrome during the treatment of pulmonary tuberculosis in an HIV1 infected patient. J Infect 2000;40:187-9. 